\title{
Anthropometric variables predicting risk of coronary artery disease in type 2 diabetics
}

\author{
Shankarappa.C ${ }^{1}$, Lingaraj Jayalakshmi ${ }^{2}$, Mohd Abdul Mateen Sidd ${ }^{3}$ \\ ${ }_{1,2,3}$ Department of physiology, Vydehi Institute Of Medical Sciences and Research Centre,, Bangalore, India
}

\begin{abstract}
:
Objective Identifying and prioritizing factors which help in predicting risk of Coronary artery Disease(CAD) in diabetics could play an important role in prevention and early treatment of coronary events. This study was done to find the association of anthropometric obesity indices with Coronary artery Disease (CAD) in type 2 diabetics and to compare the relative importance among the indices. Methods Type 2 Diabetic males on treatment since last 10 years and more than 50 years of age were included.. Subjects with history of hypertension and smoking were also included. The total sample size was 150 individuals with 94 diabetics with $C A D$ as cases and 56 diabetics without CAD as controls. The anthropometric variables were- Body Mass Index(BMI), Waist Circumference(WC), Waist Hip Ratio(WHR), Waist Height Ratio(WHtR) and Conicity Index(CI). Fasting blood glucose(FBG), HBAlC, Total cholesterol(TCho) and Triglyceride levels(TGL) were also estimated. Correlation coefficient and odds ratio was done for all parameters. Chi square was done for categorical variables. Results. Among anthropometric indices waist height ratio and waist circumference had the greatest predictive value for CAD in diabetics. The odds ratio for BMI was also slightly significant. Waist hip ratio and conicity index were not significant. Among non anthropometric risk factors duration of diabetes, smoking, hypertension and triglycerides showed significant impact on the outcome of CAD.
\end{abstract}

Key words: anthropometric variables, Coronary artery disease (CAD), diabetics, obesity indices, waist Height ratio, waist hip ratio.

\section{Introduction}

India ranks only next to China in diabetes with a prevalence of 61.3million in 2011 and an estimated increase to 101.1 million in $2030 .{ }^{(1)}$ Coronary Artery Disease(CAD)which is one of the most common causes of death in diabetics is often asymptomatic until the onset of myocardial infarction or sudden cardiac death. ${ }^{(2)}$ Regular Screening for CAD in every patient with diabetes may not be feasible or cost-effective. Consequently, factors which help in predicting risk of CAD in diabetics play an important role in prevention and early treatment of coronary events.

Obesity is a well-recognised risk factor for Type II diabetes and coronary artery disease. Several anthropometric measures are being used for screening obesity, diabetes and cardiovascular diseases. Body mass index(BMI) is used as an indicator for generalized obesity and Waist circumference(WC), waist hip ratio(WHR), Waist Height ratio(WHtR) are measures of central obesity. Central obesity is considered a better indicator of cardiovascular risk and mortality than BMI. However, the studies available to date still give no conclusive answer as to which anthropometric parameter best predicts cardiovascular events and mortality. ${ }^{(3,4)}$ A case control study from 57 countries has shown that Waist-to-hip ratio shows a graded and highly significant association with myocardial infarction risk worldwide. ${ }^{(5)}$ A metaanalysis study has shown that abdominal obesity as measured by WC and WHR is significantly associated with the risk of incident CVD events. ${ }^{(6)}$ Yet a few other evidences have given more importance for weight height ratio. ${ }^{(4)}$ Another index called conicity index is also considered as a coronary risk predictor. ${ }^{7}$ Blood Pressure, FBS, Lipid profile are also considered as physiological risk factors associated with cardiovascular diseases.

As Asians have smaller frames and different body fat composition, the relationships between anthropometric measures and physiological CHD risk factors in the Asian population are considered different from Caucasians. ${ }^{(8)}$ Studies have been done on obesity indices predicting diabetes or CAD in nondiabetics, but there are very limited studies relating anthropometric variables for CAD among diabetics. Moreover most of the studies have not included either waist hip ratio or waist height ratio. Thus this study was done to find out the association of anthropometric obesity indices and other risk factors with CAD in type 2 diabetic patients and to compare the relative importance among the indices.

\section{Methods And Materials}

The study was done in Vydehi Institute Of Medical Sciences and Research Center, Bangalore, Karnataka, India. Ethical clearance was obtained from our Institutional ethical committee and informed consent was got from all subjects. It is a case control study with a total sample size of 150 diabetic males of which 94 
were diabetics with CAD acting as cases and 56 were diabetics without CAD acting as controls. Samples were collected by random sampling method.

Type 2 Diabetic males on treatment since last 10 years and more than 50 years of age were included in the study. Subjects selected as cases were Type 2 diabetics attending medical and cardiology department of our institute and diagnosed with CAD on the basis of - definite history of Angina with documented ECG findings and under therapy, and patients who have received coronary stents or Coronary Artery Bypass grafting. Type II diabetics attending diabetic OPD with no history of CAD and after undergoing tread mill test( TMT) to rule out CAD were selected as controls. Patients with hypertension on treatment and history of smoking for more than 10 years with any number of cigarettes were also included.

Body height $(\mathrm{Ht})$ in centimeters was measured by having the subjects stand with their heels, buttocks, and heads against a wall. A flat object was placed on top of the subjects' head, and their height was marked on a tape measure affixed to the wall. Body weight was measured in kilograms $(\mathrm{kg})$ with a standard portable scale. Body weight and body height were measured with light clothes and bare feet. Waist circumference(WC) and Hip circumference $(\mathrm{HC})$ were measured according to the recommendation of the World Health Organization. ${ }^{(9)}$ For Waist Circumference, measurement was taken midpoint between the lower margin of the last palpable rib and the top of the iliac crest in a horizontal plane by the measurer sitting by the subject and fitting the tape snugly but not compressing soft tissues. Hip circumference was measured around the pelvis at the point of maximal protrusion of the buttocks. For both $\mathrm{WC}$ and $\mathrm{HC}$ subject were instructed to stand with arms at the sides, feet positioned close together, and weight evenly distributed across the feet. Circumference was measured at the end of a quiet expiration of the subject to the nearest $0.1 \mathrm{~cm}$.

From these measurements - body mass index(BMI) was calculated as body weight in kilogram divided by the square of body height in meters. Waist hip ratio(WHR) and waist height ratio(WHtR) were calculated by dividing the $\mathrm{WC}$ by $\mathrm{HC}$ and bodyHt respectively. Conicity index (CI) was constructed using the following formula: Conicity index $=$ Waist Circumference $(\mathrm{m}) /[0.109 \mathrm{X} \sqrt{ }$ \{Bodyweight $(\mathrm{kg}) /$ Height $(\mathrm{m})\}]$ where 0.109 is a constant which results from the conversion of units of volume and mass into units of length. ${ }^{(7)}$ Cut off values for BMI was $23 \mathrm{~kg} / \mathrm{m} 2$, forWC $85 \mathrm{~cm}$, for WHR 0.95 , WHeiR 0.48 , for conicity index 1.25 . The reference values for cut off were taken from studies done on Indian or Asian population. ${ }^{(7,10,11)}$

Blood samples were collected in the morning after fasting for at least $12 \mathrm{~h}$. Analysis was done in the institution central lab. Fasting plasma glucose, serum total cholesterol, triglyceride were measured using - timed endpoint method and hemoglobin Alc concentration by - turbidimetric immune inhibition method using Unicell dxc 600 and a synchron 5 pro clinical chemistry system from Beckman coulter.

SPSS- 16.0 package was used for statistical analysis. Pearson's correlation coefficient and odds ratio by logistic regression analysis were done. Chi square test was done for categorical variables smoking and hypertension. The confidence interval was at $95 \%$ and $\mathrm{P}$ value was considered significant when $<0.05$.

\section{Results}

The mean values for all continuous variables are shown in group statistics table.(TABLE1). Results of Correlation coefficient ( $\mathrm{r}$ value) showing strength of association between the anthropometric variables (TABLE 2 AND 3 ) showed that in cases a good correlation- ( $r=0.6$ to 0.8 )was seen between waist, waist height ratio and conicity index. In controls good correlation was seen between waist and conicity index. There was no correlation with waist height ratio as seen in cases. Mild correlation ( $r=0.4$ to 0.6$)$ was seen with waist hip ratio and waist height ratio in cases. Mild correlation was also seen with waist hip ratio, waist and conicity in both cases and controls. The $r$ value between the non anthropometric variables was very less showing a poor strength of association in both cases and controls.

Odds ratio showing the risk of outcome was done for the anthropometric indices with their specific cut off points. (GRAPH) . Odds ratio was high for waist height ratio and waist with greater significance for waist height ratio. The odds ratio for BMI was also slightly significant. Waist hip ratio and conicity index were not significant. Odds ratio for non anthropometric variables showed significant impact of duration of diabetes $(\mathrm{p}=0.001)$,smoking $(\mathrm{p}=0.024), \mathrm{BP}(\mathrm{p}=0.001)$ and triglycerides $(\mathrm{p}=0.004)$ on the outcome of CAD.

Chisquare test also showed that the number of smokers were $67.1 \%$ in cases and $48.2 \%$ in controls $(\mathrm{p}=0.023)$ and hypertensives were $62.8 \%$ in cases when compared to $33.9 \%$ in controls $(\mathrm{p}=0.001)$ 


\section{Figures And Tables}

TABLE 1 -group statistics showing mean values of continuous variables

\begin{tabular}{|c|c|c|c|c|c|}
\hline \multicolumn{2}{|c|}{$\begin{array}{c}\text { Variables \& Group } \\
\text { GP - 1(cases) with CAD Gp -2 (controls) } \\
\text { without CAD }\end{array}$} & $\mathrm{N}$ & Mean & Std. Deviation & Std. Error Mean \\
\hline \multirow[t]{2}{*}{ AGE } & 1 & 94 & 55.63 & 4.192 & .432 \\
\hline & 2 & 56 & 54.62 & 3.404 & .455 \\
\hline \multirow{2}{*}{ Diabetes Duration } & 1 & 94 & 13.30 & 1.671 & .172 \\
\hline & 2 & 56 & 12.36 & 1.271 & .170 \\
\hline \multirow{2}{*}{ Waist $\{\mathrm{Cm}\}$} & 1 & 94 & 84.70 & 2.471 & .255 \\
\hline & 2 & 56 & 82.91 & 3.123 & .417 \\
\hline \multirow{2}{*}{ Hip $\{\mathrm{Cm}\}$} & 1 & 94 & 87.49 & 2.266 & .234 \\
\hline & 2 & 56 & 85.70 & 2.935 & .392 \\
\hline \multirow{2}{*}{ Height $\{\mathrm{Cm}\}$} & 1 & 94 & 170.91 & 2.734 & .282 \\
\hline & 2 & 56 & 170.70 & 2.783 & .372 \\
\hline \multirow{2}{*}{ Weight $\{\mathrm{Kg}\}$} & 1 & 94 & 66.86 & 3.294 & .340 \\
\hline & 2 & 56 & 65.77 & 2.717 & .363 \\
\hline \multirow{2}{*}{ Waist-To-Hip Ratio } & 1 & 94 & .9671 & .01206 & .00124 \\
\hline & 2 & 56 & .9634 & .00978 & .00131 \\
\hline \multirow{2}{*}{ Waist-To-Height Ratio } & 1 & 94 & .495 & .0164 & .0017 \\
\hline & 2 & 56 & .494 & .0667 & .0089 \\
\hline \multirow{2}{*}{ Body mass Index } & 1 & 94 & 23.037 & 1.1347 & .1170 \\
\hline & 2 & 56 & 22.614 & 1.2579 & .1681 \\
\hline \multirow{2}{*}{ Conicity Index } & 1 & 94 & 1.2432 & 0.04417 & 0.00456 \\
\hline & 2 & 56 & 1.2212 & 0.05504 & 0.00736 \\
\hline \multirow{2}{*}{ Fasting Plasma Glucose (mg/dl) } & 1 & 94 & 144.88 & 9.315 & .961 \\
\hline & 2 & 56 & 143.61 & 9.576 & 1.280 \\
\hline \multirow{2}{*}{ Hbalc(\%) } & 1 & 94 & 7.754 & .1349 & .0139 \\
\hline & 2 & 56 & 7.743 & .1142 & .0153 \\
\hline \multirow{2}{*}{ Total Cholesterol(mg/dl) } & 1 & 94 & 187.14 & 12.221 & 1.260 \\
\hline & 2 & 56 & 187.73 & 11.816 & 1.579 \\
\hline \multirow{2}{*}{ Triglyceride(mg/dl) } & 1 & 94 & 164.86 & 10.493 & 1.082 \\
\hline & 2 & 56 & 160.09 & 7.227 & .966 \\
\hline
\end{tabular}

TABLE 2 -Correlation coefficient ( $\mathrm{r}$ value) among cases showing strength of association between the variables

\begin{tabular}{|llllll|}
\hline & Waist & $\begin{array}{l}\text { Waist hip } \\
\text { ratio }\end{array}$ & $\begin{array}{l}\text { Waist height } \\
\text { ratio }\end{array}$ & BMI & Conicity index \\
\hline Waist & - & 0.536 & 0.797 & 0.139 & 0.746 \\
\hline Waist hip ratio & - & - & 0.445 & 0.022 & 0.431 \\
\hline Waist height ratio & - & - & - & 0.188 & 0.688 \\
\hline Body Mass Index & - & - & - & - & 0.303 \\
\hline
\end{tabular}

TABLE 3 -Correlation coefficient ( $r$ value) among controls

\begin{tabular}{|c|c|c|c|c|c|}
\hline & \multicolumn{2}{|c|}{ Waist } & \multirow{2}{*}{$\begin{array}{l}\text { Waist height } \\
\text { ratio } \\
0.288\end{array}$} & \multirow{2}{*}{$\begin{array}{l}\text { BMI } \\
0.146\end{array}$} & \multirow{2}{*}{$\begin{array}{l}\text { Conicity index } \\
0.755\end{array}$} \\
\hline Waist & - & 0.594 & & & \\
\hline Waist hip ratio & - & - & 0.117 & 0.010 & 0.448 \\
\hline Waist height ratio & - & - & - & 0.122 & 0.171 \\
\hline Body Mass Index & - & - & - & - & 0.330 \\
\hline
\end{tabular}




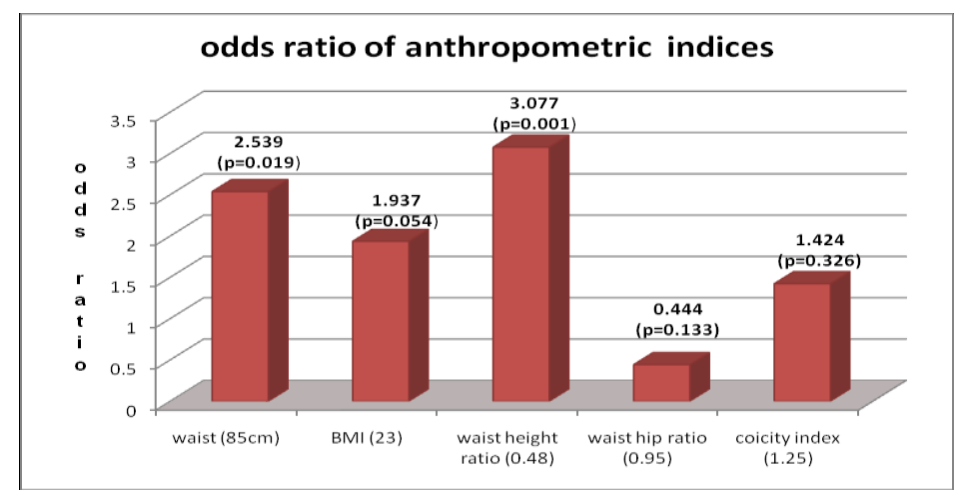

\section{Discussion}

Anthropometric obesity indices and physiological risk factors were studied in 94 male diabetics with $\mathrm{CAD}$ and 56 male diabetics without CAD.

As there were very less reports relating anthropometric indices on CAD in diabetics our literature review included studies associating anthropometric measures with CAD in non diabetics also. A study from 57 countries and another metaanalysis study have reported significant association of WHR and WC with cardiovascular events. $\left({ }^{5,6}\right)$ But in these studies WHtR was not considered. A systematic review of seventyeight studies exploring waist-to-height ratio (WHtR) and waist circumference (WC) or BMI, showed that WHtR and WC were significant predictors of cardiometabolic outcomes more often than BMI. ${ }^{(12)}$ But in this review WHR was not considered. In an Indian study on railway employees the results state that when compared to all other anthropometric variables, WHtR was statistically significantly associated with a majority of coronary artery risk factors. ${ }^{(13)}$

A study done similar to our study on anthropometric indices and CAD in Taiwanese type 2 diabetic patients, showed that among the four indices of obesity (WC, WHR, WHtR, and BMI) only WHtR shows consistent and independent association with $\mathrm{CAD}$ with $\mathrm{WC}$ as an additional risk factor for men and $\mathrm{BMI}$ for women. ${ }^{(4)}$ In Comparison results of the present study showed that waist height ratio and waist circumference have the greatest predictive value for $C A D$ in diabetics with a significance of $p=0.001$ and 0.019 respectively which is similar to the Taiwan study, but in our study BMI also was slightly significant with $\mathrm{p}=0.05$ even though all subjects were males which differs from the other study which had reported significance for BMI only in females.

An Indian study on CAD in both diabetics and non diabetics showed that BMI, WHR and WC (WHtR was not included) had a significant correlation with CAD in nondiabetic subjects but were not correlated with $\mathrm{CAD}$ in diabetic patients thus implying that parameters correlating with CAD are different for diabetics and non diabetics. ${ }^{(14)}$ But this is again controversial as WC was shown as significant for CAD in studies done on non diabetics as well as in the Taiwan study done on diabetics and in the present study.

A Framingham Heart Study comparing Conicity index and BMI on predicting coronary heart disease reports that $\mathrm{BMI}$ is a better marker than CI for predicting CHD incidence and mortality. ${ }^{(15)}$ In our study even though the strength of association(by pearsons correlation) was good in cases between WHtR and CI there was no effect on the outcome as seen by odds ratio. The correlation seen with waist and conicity Index in both cases and controls shows that these parameters can be used as indices for diabetes but may not be useful in predicting CAD in diabetics.

The cut off values for anthropometric measurements have been shown to be lower in Asian countries than their European and western counterparts. In this study the cut off for Waist hip ratio was higher (0.95) than that considered as standard for Indians (0.90) as none of the values in this study were less than 0.90 (lower cut off). This high ratio could be due to the reduced hip circumference seen in most of the participants(both cases and controls).

The non anthropometric variables in diabetics which showed better predictability of CAD in this study were duration of diabetes, smoking, hypertension and triglyceride. Other studies have shown age and increased glucose levels also as a risk factor for CAD. ${ }^{(16)}$ But in this study their mean values were almost the same between cases and controls and thus showed no predictability.

\section{Limitations Of This Study}

Even though the results are significant and the study has clinical relevance in the Indian context there are some limitations. The sample size is small and the results would be more conclusive with a larger sample size. Although the lesser number of controls than cases does not affect the statistical results an equal number of 
cases and controls would have been better. Also the treatment history of hypertensive patients was not considered in this study.

\section{Conclusion}

Diabetics of long duration, even though their plasma glucose and HBA1C are fairly under control are prone for cardiovascular complications. Hence it is essential to identify risk factors predicting risk of CAD in diabetics. For the Indian population Waist Height Ratio and Waist circumference can be considered as better indices to predict the risk of CAD in diabetics. The non significance of Waist Hip Ratio in this study need to be further evaluated by studies comparing both WHR and WHtR in a larger population.

\section{Acknowledgements}

Sincere gratitude to our management and advisor for financial support and the department of cardiology and medicine for their cooperation and help in selecting the subjects.

\section{References}

[1]. International Diabetes Federation. IDF Diabetes Atlas, 5th edn. Brussels, Belgium: International Diabetes Federation, 2011. Available from http://www.idf.org/diabetesatlas.

[2]. Sandeep Chopra, Soumia Peter._Screening for coronary artery disease in patients with type 2 diabetes mellitus: An evidence-based review. Indian J Endocrinol Metab. 2012 Jan-Feb; 16(1): 94-101.

[3]. Harald J. Schneider, Nele Friedrich, Jens Klotsche,Lars Pieper, Matthias Nauck, Ulrich John, et al. The Predictive Value of Different Measures of Obesity for Incident Cardiovascular Events and Mortality. The Journal of Clinical Endocrinology \& Metabolism. 2010 April1;95(4):1777-1785.

[4]. Chin-Hsiao Tseng. Waist-to-height Ratio and Coronary Artery Disease in Taiwanese Type 2 Diabetic Patients. Obesity .2008; 16 (12): 2754-2759.

[5]. Salim Yusuf, Steven Hawken, Stephanie Ônpuu, Leonelo Bautista, Maria Grazia Franzosi, Patrick Commerford etal. Obesity and the risk of myocardial infarction in 27000 participants from 52 countries: a case-control study. Lancet. 2005Nov; 366: 1640-49.

[6]. Lawrence de Koning, Anwar T. Merchant, Janice Pogue and Sonia S. Anand. Waist circumference and waist-to-hip ratio as predictors of cardiovascular events: meta-regression analysis of prospective studies. European Heart Journal.2007; 28: 850-856.

[7]. Meerjady Sabrina Flora, Cgn Mascie-Taylor, Mahmudur Rahman . Conicity index of adult Bangladeshi population and their sociodemographic characteristics. ibrahim med. coll. j. 2009; 3(1): 1-8

[8]. Sarah Choi, Edwin Tan. Anthropometric measures and lipid CHD risk factors in Korean Immigrants with Type 2 Diabetes.J Cardiovasc Nurs. 2011 Sep-Oct; 26(5): 414-422.

[9]. Waist Circumference and Waist-Hip Ratio .Report of a WHO Expert Consultation Geneva, 8-11 December 2008. Available from http://whqlibdoc.who.int/publications/2011/9789241501491_eng.pdf.

[10]. Chamukuttan Snehalatha,Vijay Viswanathan,Ambady Ramachandran. Cutoff Values for Normal Anthropometric Variables in Asian Indian Adults Diabetes Care. 2003; 26:1380-1384.

[11]. Meerjady Sabrina Flora, CGN Mascie-Taylor, Mahmudur Rahman. Waist-To-Height Ratio And Socio-Demographic Characteristics Of Bangladeshi Adults Ibrahim Med. Coll. J. 2010; 4(2): 49-58.

[12]. Lucy M. Browning, Shiun Dong Hsieh and Margaret Ashwell. A systematic review of waist-to-height ratio as a screening tool for the prediction of cardiovascular disease and diabetes. Nutrition Research Reviews .2010; 23: 247-269.

[13]. Virendra C. Patil, G. P. Parale, P. M. Kulkarni, and Harsha V. Patil. Relation of anthropometric variables to coronary artery disease risk factors. Indian J Endocrinol Metab. 2011 Jan-Mar; 15(1): 31-37.

[14]. Harsimran Kaur, Sharda Sidhu and Vasudha Sambyal. Association of Obesity Indices with Type 2 Diabetes Mellitus and Coronary Artery Disease. J Hum Ecol.2010;29(3): 185-190.

[15]. K S Kim, W L Owen, D Williams, L L Adams-Campbell. A comparison between BMI and Conicity index on predicting coronary heart disease: the Framingham Heart Study. Annals of Epidemiology 2000nov; 10(7):424-31.

[16]. António Vaz Carneiro. A Doença Coronária na Diabetes Mellitus.Factores de risco e Epidemiologia. Rev Port Cardiol 2004;23 (10) :1359-1366 